\title{
Spontaneous development of retinal ischaemia and rubeosis in eyes with retinal racemose angioma
}

\author{
Philip A Bloom, Alistair Laidlaw, David L Easty
}

Department of Ophthalmology, Bristo Eye Hospital, Lower Maudlin Street, Bristol BS1 2LX

P A Bloom

D A H Laidlaw

D L Easty

Correspondence to:

Philip A Bloom, The Western

Ophthalmic Hospital,

Marylebone Road, London

NW1 5 YE.

Accepted for publication

17 September 1992

Figure 1 Case 1. Montage fundus photograph showing the retinal racemose angioma before the development of retinal ischaemia.
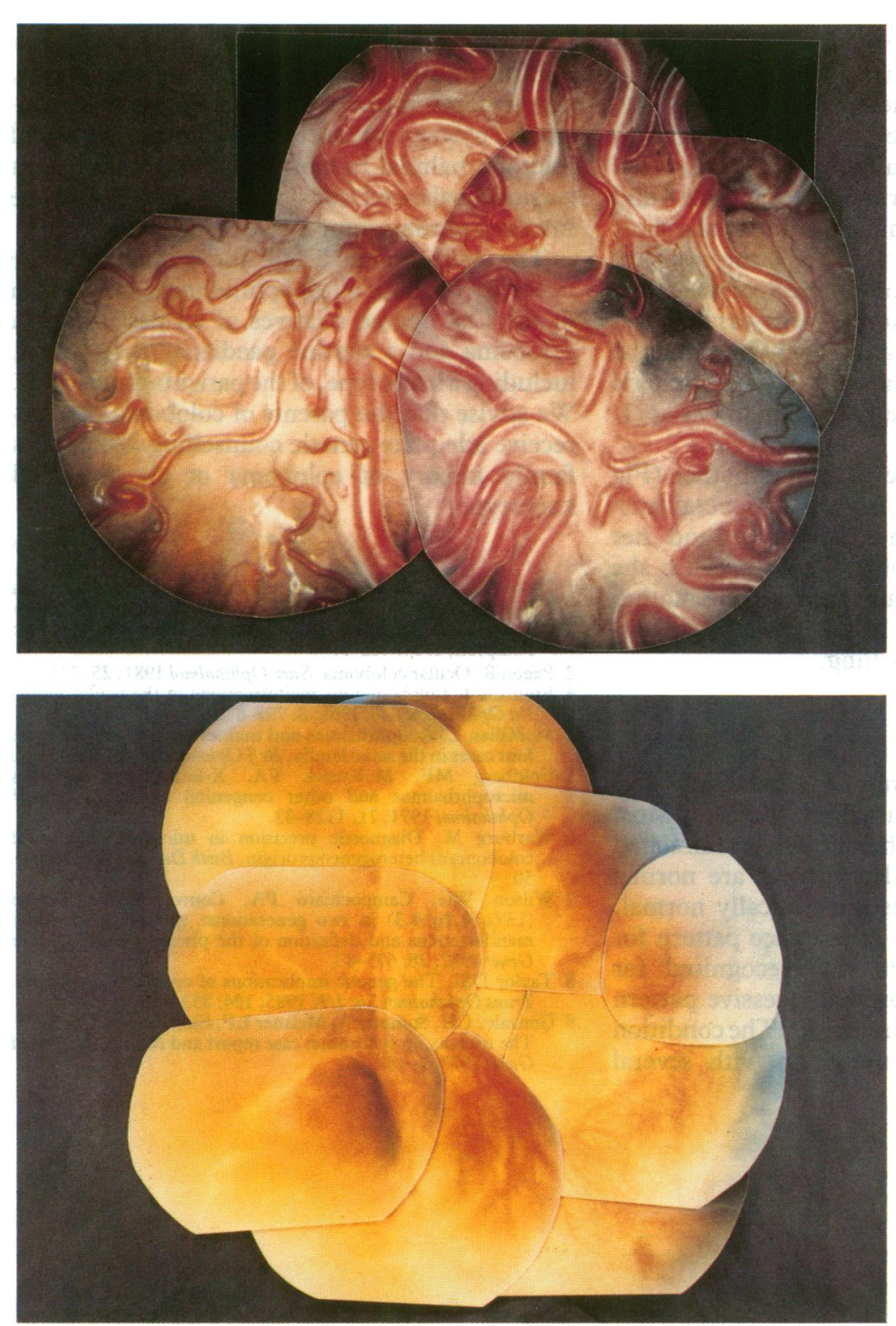

Figure 2 Case 1. Montage fundus photograph after the development of retinal ischaemia and retinal detachment.

\begin{abstract}
Two cases are described of spontaneous vascular closure occurring in eyes with arteriovenous malformations, causing visual loss due to retinal ischaemia and rubeosis iridis.

(BrF Ophthalmol 1993; 77: 124-125)
\end{abstract}

\section{Case reports}

CASE 1

A 7-year-old boy was seen at Bristol Eye Hospital in 1981 with a lifelong history of an intermittent left divergent squint. Best corrected visual acuity in the left eye was $6 / 24$. This was found to be due to a retinal racemose angioma, with relative sparing of the macula (Fig 1).

At the age of 13 years he re-presented as an emergency because of pain in the left eye associated with a sudden reduction of visual acuity, measured as $3 / 60$. He was found to have a left relative afferent pupil defect, rubeotic glaucoma, and the fundal appearance of widespread ischaemia and vascular closure. He subsequently developed retinal neovascularisation and a traction detachment of the retina (Fig 2). Computed tomography (CT) examination revealed no evidence of orbital or intracerebral angiomas.

\section{CASE 2}

An 8-year-old girl was seen at Moorfields Eye Hospital in 1980, having become aware of poor vision in the right eye on the previous day. Visual acuity with the right eye was hand movements, there was a right relative afferent pupillary defect, and a partial hyphaema. Large telangiectactic vessels were seen in the superior fundus. Over the next 24 hours further bleeding occurred into the anterior chamber, the eye became painful, the intraocular pressure rose to $46 \mathrm{~mm} \mathrm{Hg}$, and rubeosis was visible on the superior iris. Despite intensive medical therapy, the pressure could not be controlled and a Molteno tube was inserted 8 days later. Thereafter the hyphaema cleared rapidly, the rubeosis resolved, the eye became comfortable and a clear fundal view was obtained.

An arteriovenous malformation was observed centred on the optic nerve head with large, closed, opaque blood vessels in the peripheral fundus (Fig 3), an impression confirmed by fluorescein angiography (Fig 4). Carotid angiography revealed the presence of a small arteriovenous malformation in the region of the optic nerve in the posterior orbit, but this did not extend into the cranial cavity. Apart from one brief episode of ocular hypertension soon after surgery the intraocular pressure remained normal. When last seen, the visual acuity with the right eye was $1 / 60$, the right visual field was severely restricted, and the intraocular pressure was normal.

\section{Comment}

Congenital unilateral arteriovenous malformations of the retinal vasculature usually consist of fully developed vessels and are usually confined to the posterior pole.' The lesions have been reported to remain static in size,' to enlarge gradually, ${ }^{2}$ and spontaneously to regress. ${ }^{3}$ Vision may be affected directly due to macular involvement, ${ }^{+}$by secondary retinal cystic 


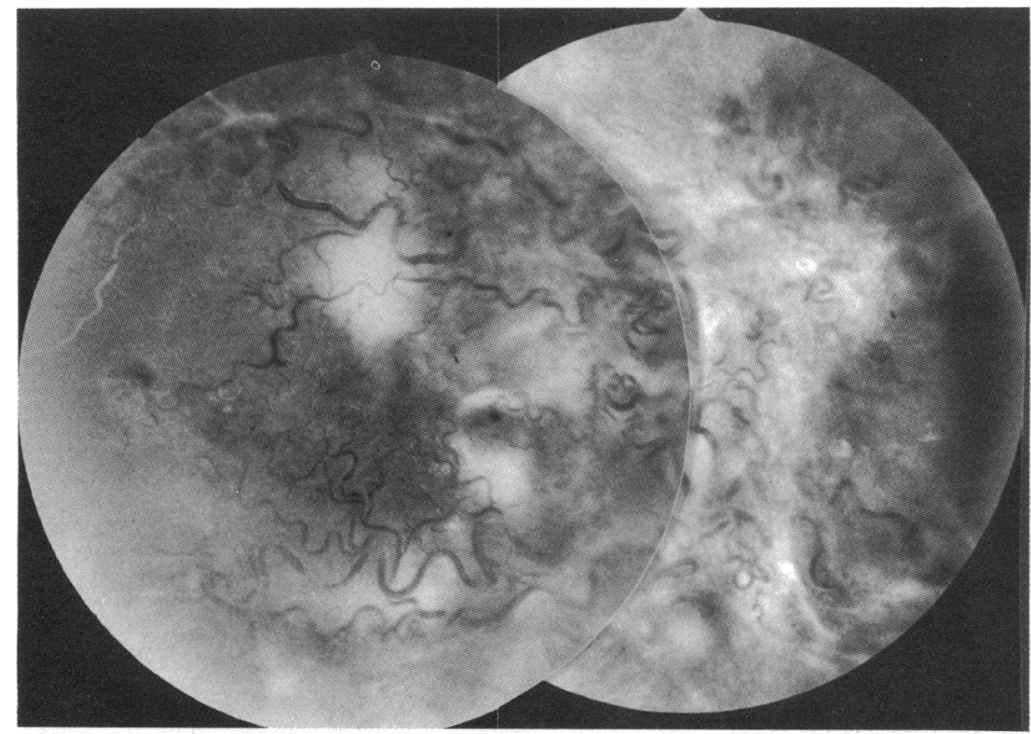

Figure 3 Case 2. Montage fundus photograph showing the retinal racemose angioma at the posterior pole, and closed peripheral vessels.

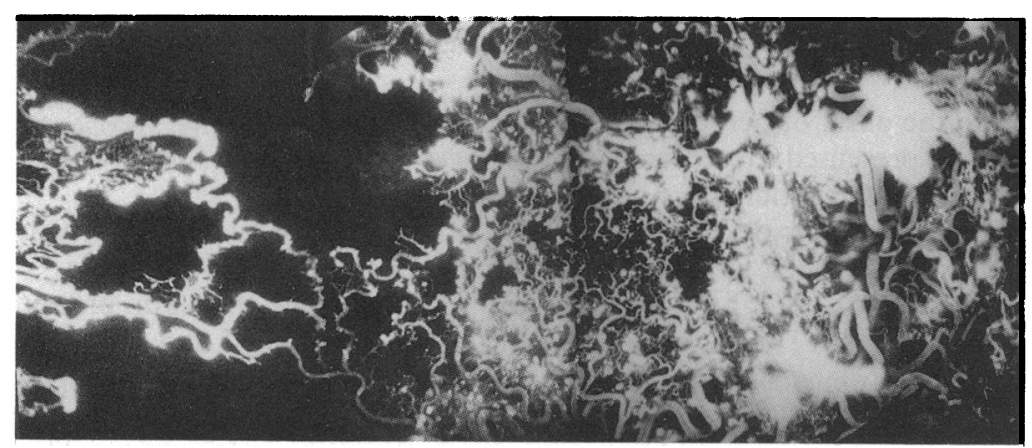

Figure 4 Case 2. Montage fundus fluorescein angiogram showing leakage of dye from the arteriovenous malformation, extensive loss of peripheral retinal perfusion and staining of peripheral fundal vessels. change, ${ }^{5}$ or by producing haemorrhage or exudation. ${ }^{6}$ Retinal arteriovenous malformations may be associated with arteriovenous malformations of the optic nerve and midbrain, ${ }^{7}$ which carries the eponym of the Wyburn-Mason syndrome, or with more widespread involvement in the territories of the internal and external carotid arteries. ${ }^{89}$

To our knowledge there is only one reported case in the literature similar to those that we present. ${ }^{2}$ In this case, a 4-year-old girl with Wyburn-Mason syndrome developed retinal ischaemia and rubeosis iridis spontaneously, in an eye with a racemose angioma that had been previously documented to vary in size and that remained perfused following the development of retinal ischaemia. In the cases we report, little is known of the behaviour of the lesion before the identification of visual loss. In both cases the patients claimed sudden disturbance of vision, although the presence of rubeosis suggests that retinal ischaemia existed for some time before presentation. It is possible that there was peripheral retinal non-perfusion before loss of macular function. In both cases the perfusion deficit involved both the normal and abnormal retinal vasculature.

Rubeosis associated with retinal racemose angiomas has been postulated by Trabouls ${ }^{10}$ to be due to one of the following mechanisms: (i) chronic ischaemia of the retina owing to 'steal' of its blood supply by the enlarged malformation; (ii) partial thrombosis of the arteriovenous malformation with resulting retinal ischaemia (this presumes the affected retina to be supplied, at least in part, by the portion of the malformation that thrombosed; (iii) compression of the retinal venous outflow at the optic nerve head by the enlarging arteries supplying the malformation resulting in a retinal vein occlusion. The last explanation was favoured by Traboulsi, although Tomsak believed that arterial occlusion was the primary event. ${ }^{11}$

The aetiology of the ischaemia in the two cases reported above is unclear. In the first case the malformation closed as well as the retinal circulation, so vascular steal alone is an unlikely explanation. It is possible that partial thrombosis occurred at a time of circulatory stasis within the lesion, while the pattern of blood flow was changing. In order to have given rise to the observed widespread ischaemia, it is evident that this thrombosis would have occurred near the origin of the malformation at the optic nerve head. Occlusion of the retinal venous circulation with consequent vascular closure and rubeosis, as may be seen in a central retinal vein occlusion, cannot be discounted although there is little to suggest that this occurred.

We thank Professor A C Bird, Department of Clinical Ophthalmology, Moorfields Eye Hospital for his permission to report the second case, and for his help with the manuscript.

We are grateful to Mrs $G$ Bennerson of Bristol Eye Hospital for Figures 1 and 2, and to Professor Bird and the Medical Illustration Department of Moorfields Eye Hospital for Figures 3 and 4.

1 Rundles WZ, Falls HF. Congenital arteriovenous (racemose) aneurysm of the retina. Arch Ophthalmol 1951; 46: 408-18.

2 Effron L, Zakov ZN, Tomsak RL. Neovascular glaucoma as a complication of the Wyburn-Mason syndrome. F Clin Neuro Comphication of the Wybu $1985 ; 5: 95-8$.

3 Dekking HM. Arteriovenous aneurysm of the retina with spontaneous regression. Ophthalmologica 1955; 130: 113-5.

4 Font RL, Ferry AP. The phakomatoses. Int Ophthalmol Clinics 1972; 12: 1-50.

5 Archer DB, Deutman A, Ernest JT, Krill AE. Arteriovenous communications of the retina. Am $\mathcal{F}$ Ophthalmol 1973; 75: 224-41.

6 Bernth-Peterson P. Racemose haemangioma of the retina: report of three cases with long term follow up. Acta Ophthalmol $(K b h) 1979 ; 57: 669-78$.

7 Wyburn-Mason R. Arteriovenous aneurysm of mid-brain and retina, facial naevi and mental changes. Brain 1943; 66: 163 203 .

8 Tamaki N, Fujita K. Yamashita H. Multiple arteriovenous malformations involving the scalp, dura, retina, cerebrum and posterior fossa. F Neurosurg 1971; 34: 95-8.

9 Theron J, Newton TH, Hoyt WF. Unilateral retinocephalic vascular malformations. Neuroradiology 1974; 7: 185-96.

10 Traboulsi EI. Neovascular glaucoma and ischemia. $\mathcal{f}$ Clin Neuro Ophthalmol 1986; 6: 126.

11 Tomsak RL. Neovascular glaucoma and ischemia. 7 Clin Neuro Ophthalmol 1986; 6: 127. 